\title{
Role of Various Spacer Materials on Magnetic Properties of CoNbZr Amorphous Multilayered Thin Films
}

\author{
W. J. Choe, T. D. Lee*, C. H. Hwang and Y. S. Park \\ Dept. of Metall. Eng., Yonsei University, Seoul 120-749, Korea \\ * Div. of Metals, Korea Institute of Science and Technology, Seoul 136-791, Korea
}

\begin{abstract}
In recent years, multilayered Co base amorphous thin films have been extensively studicd to improve permeability at high frequency range and to control the closure domain structure in a narrow tipped region. In the present work $\mathrm{CoNbZr}$ multilayered films were made using electrically non conductive $\mathrm{SiO}_{2}$ and conductive $\mathrm{Cu}$ and carbon spacers. Effects of $\mathrm{SiO}_{2}$ thickness on coercivity and permeability wcre studied. Although DC hysteresis showed very similar behavior, multilayered $\mathrm{CoNbZr} /$ conductive spacer films showed lower permeability than $\mathrm{CoNbZr} / \mathrm{SiO}_{2}$ spacer films throughout the whole frequency range. It is concluded this lower permeability behavior is not related to eddy current loss.
\end{abstract}

\section{Introduction}

There have been many attempts to improve soft magnetic properties of thin films as high coercivity materials are used for high density recording. Besides permalloy and sendust films, such soft magnetic films as Co base amorphous, iron base nanocrystalline and $\mathrm{Fe}$ nitrides films are intensively being studied.

Multilayer films of two or more magnetic films separated by thin non magnetic interlayers are known to have lower coercivity and high permeability response of frequency than monolithic films of the same thickness. However, as spacer materials, non magnetic and non conductive materials such as $\mathrm{SiO}_{2}^{(1)}, \mathrm{Al}_{2} \mathrm{O}_{3}^{(2)}$ and $\mathrm{AlN}^{(3)}$ have been studied. In this work, the non magnetic and conductive $\mathrm{Cu}$ and carbon films as well as $\mathrm{SiO}_{2}$ films were used as spacer materials in $\mathrm{Co}_{92} \mathrm{Nb}_{5.6} \mathrm{Zr}_{2.4}$ multilayered films. Effects of $\mathrm{SiO}_{2}$ thickness on magnetic properties of the films were studied. Also the effect of conductive layers on permeability is studied.

\section{Experimental Procedure}

$\mathrm{Co}_{92} \mathrm{Nb}_{5.6} \mathrm{Zr}_{2.4}$ amorphous films were formcd on $800 \mathrm{~A}$ thick $\mathrm{SiO}_{2}$ film pre-deposited commercial slide glass substrates by an if magnetron sputtering unit. Deposition rate of $\mathrm{CoNbZr}$ was $160 \mathrm{~A} / \mathrm{min}$. For multilayered films, $\mathrm{SiO}_{2}, \mathrm{Cu}$ and carbon spacer materials were used. When $\mathrm{SiO}_{2}$ was sputtered using a $\mathrm{SiO}_{2}$ target, $2 \%$ of $\mathrm{O}_{2}$ was added to the sputtering gas to ensure the formation of sufficiently oxidized film.

Total thickness of CoNbZr film was kept to $0.3 \mu \mathrm{m}$ regardless of film stacking mode. The substrates were cleaned, heated to $150{ }^{\circ} \mathrm{C}$ in vacuum and then cooled down to room temperature through water circulation in the substrate holder prior to deposition. Ferrite magnets were placed beneath the substrate holder to align the easy axis of the as-sputtered films.

Post heat treatments were done in the temperature range from 200 to $350{ }^{\circ} \mathrm{C}$ for $2 \mathrm{hrs}$ in a rotating field annealing(RFA) furnace. The specimens were rotated at a speed of $30 \mathrm{rpm}$ in 
a magnetic field of $500 \mathrm{Oe}$. The degree of the vacuum were better than $1 \times 10^{-5}$ torr.

Magnetic properties were measured by a VSM. Effective permeability was measured with a impedance analyzer.

\section{Experimental Results}

Chemical composition of the film analyzed by ICP method was $\mathrm{Co}_{92} \mathrm{Nb}_{5.6} \mathrm{Zr}_{2.4}$, while that of the target was $\mathrm{Co}_{84} \mathrm{Nb}_{11.5} \mathrm{Zr}_{4.5}$. Saturation magnetization of the film was 11.5 KGauss, which is very close to the calculated $\mathrm{Ms}$ value of Co alloy with 2.4 at\% $\mathrm{Zr}$ and $5.6 \quad \mathrm{at} \% \quad \mathrm{Nb}$ considering the simple law of mixture.

Fig. 1 shows coercivity change of $\mathrm{CoNbZr}$ single layers in as-deposited monolithic films and multilayered films where 50 and $20 \mathrm{~A} \quad \mathrm{SiO}_{2}$ spacers were used. As previously reported, coercivity of the films decreases with decreasing the spacer thickness. This indicates magnetic interaction between the magnetic layers becomes stronger as the thickness of the spacer becomes thinner. Coercivity of multilayered films of total 3000 A thick showed little decrease compared to that of the monolithic layer of the same thickness. However, the multilayered film of total $1500 \mathrm{~A}$ thick showed substantial decrease in coercivity compared to the monolithic layer of the same thickness. Coercivity dropped from 1.3 Oe to 0.5 - 0.8 Oe by the multilayer scheme. This must be due to larger surface roughness contribution on domain wall pinning ${ }^{(3)}$ and higher spin dispersion contribution in the thinner monolithic films, although magnetostatic interaction between the magnetic layers of different thickness are not significantly changed as appeared in Fig. 1.

Variation of coercivity in hard direction as the temperature of RFA increases is appeared in Fig. 2. There is no significant change of cocrcivity. This indicates relief of residual stress and different degree of local easy axis dispersion

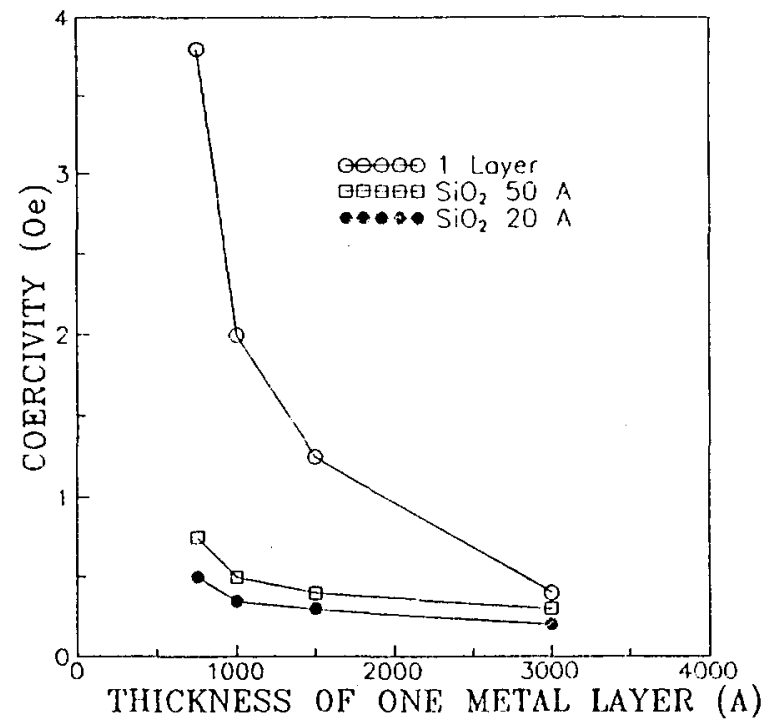

Fig. 1 Dependence of $\mathrm{Hc}$ on the thickness of $\mathrm{CoNbZr}$ layer and $\mathrm{SiO}_{2}$ layer

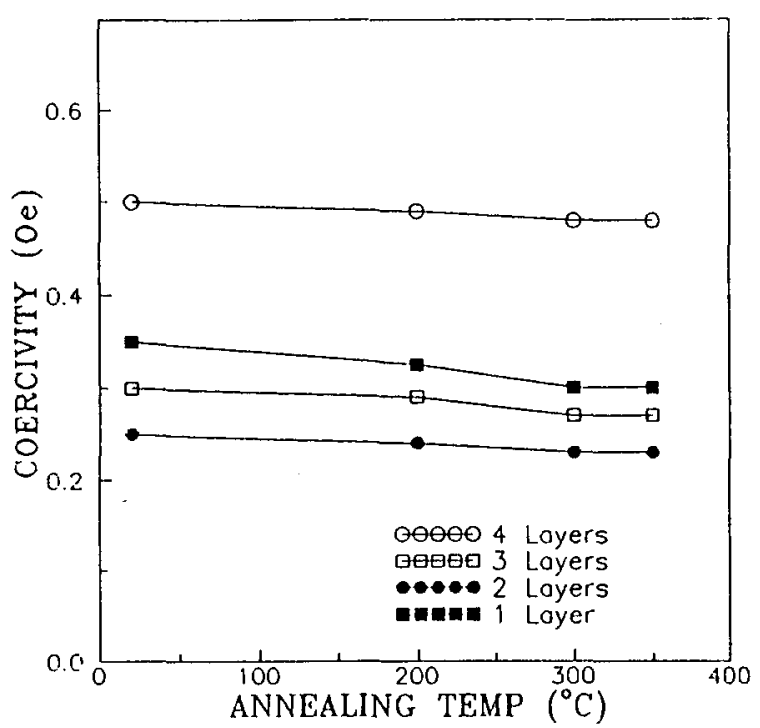

Fig. 2 Variation of $\mathrm{Hc}$ as a function of annealing temperature (After RFA 2hr, hard direction, $\mathrm{SiO}_{2} 20 \mathrm{~A}$ )

do not contribute significantly on coercivity of the film. However, as shown in Fig. 3, anisotropy fields measured in hard and soft directions are converging to a same value when they were annealed at $350{ }^{\circ} \mathrm{C}$.

Frequency dependence of effective permeability of as-deposited films measured in hard direction is 


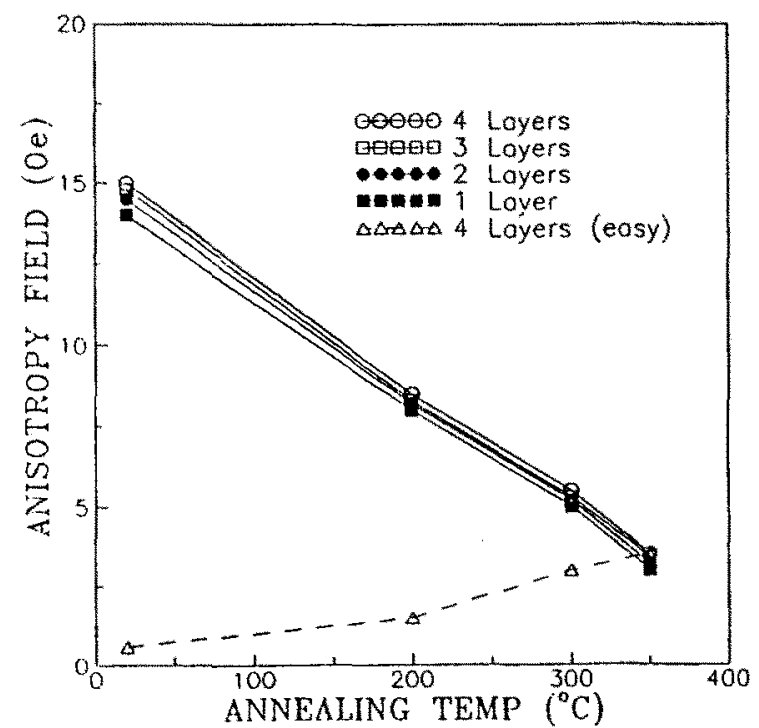

Fig. 3 Variation of effective anisotropy field as a function of annealing temperature (After RFA 2hr, hard direction, $\mathrm{SiO}_{2} 20 \mathrm{~A}$ )

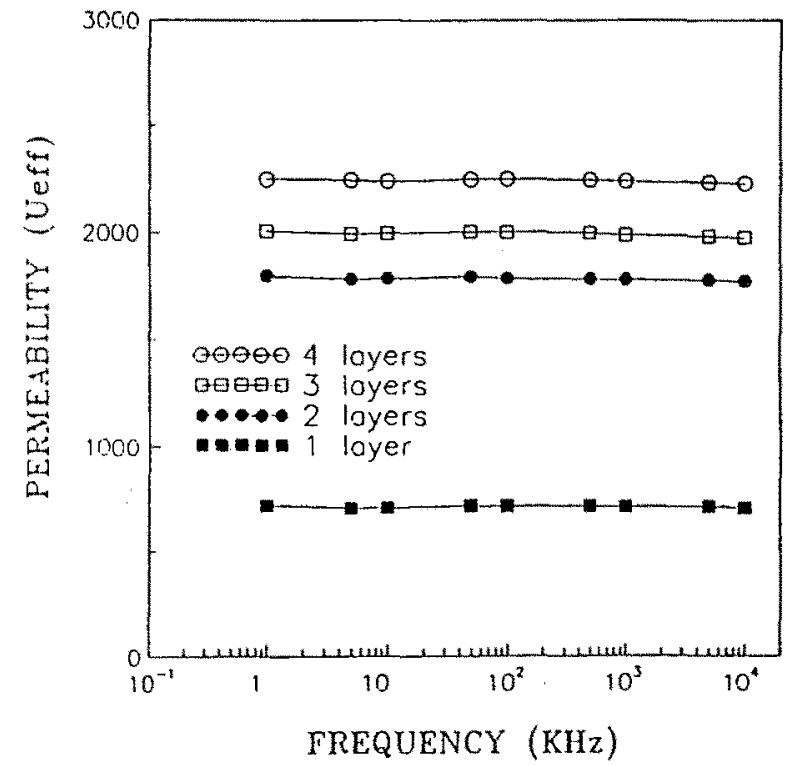

Fig. 4 Dependence of effective permeability on frequency (As-deposited, hard direction, $\mathrm{SiO}_{2} 20 \mathrm{~A}, \mathrm{Hex}=600 \mathrm{mOe}$ )

appeared in Fig. 4 and 5. For the same total magnetic layer thickness, effective permeability increases with increasing number of stacks and with decreasing spacer thickness. This is again

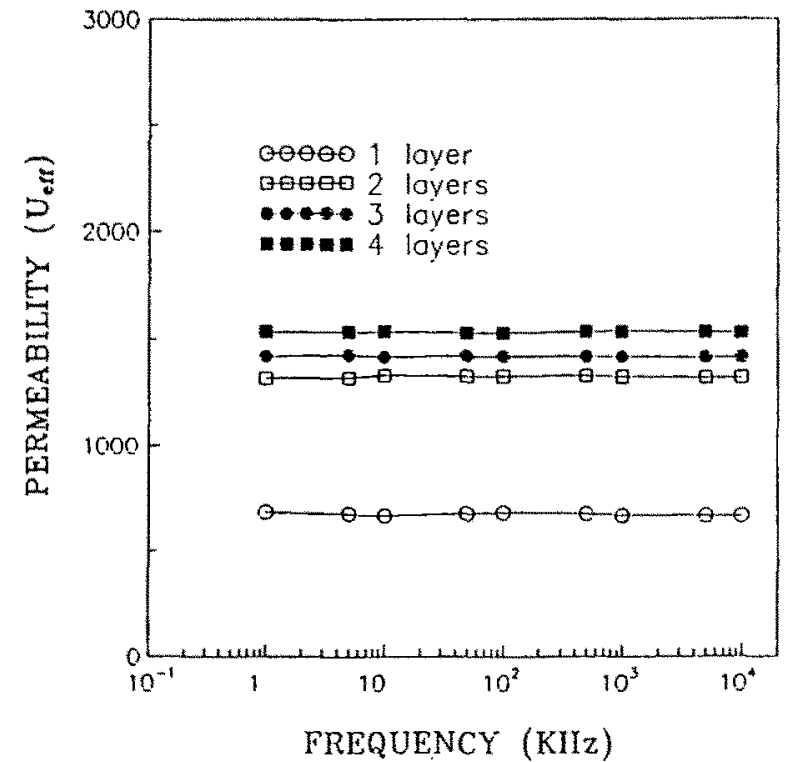

Fig. 5 Dependence of effective permeability on frequency (As-deposited, hard direction, $\mathrm{SiO}_{2} 250 \mathrm{~A}, \mathrm{Hex}=600 \mathrm{mOe}$ )

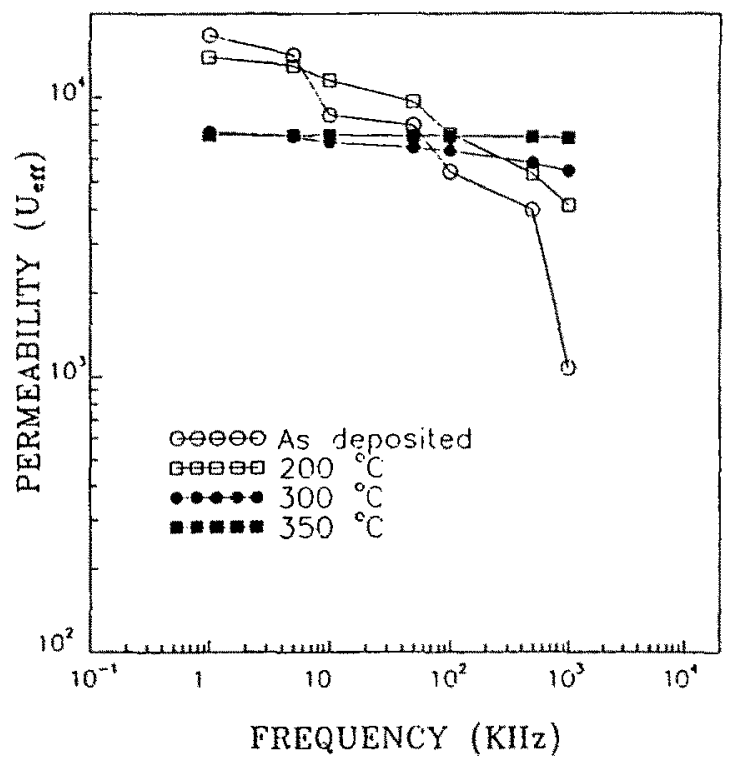

Fig. 6 Dependence of effective permeability on frequency (After RFA $2 \mathrm{hr}$, no field, $\mathrm{SiO}_{2}$ $20 \mathrm{~A}, 4$ layers, $\mathrm{Hex}=600 \mathrm{mOe}$ )

indicating stronger magnetic interactions are directly related to the permeability response.

Fig. 6 shows effective permeability change of the four layered specimens in hard direction with 
increasing annealing temperature. Effective permeability increases with increasing annealing temperature and saturates at around $350{ }^{\circ} \mathrm{C}$ annealing. This behavior of effective permeability is very closely related to decrease of $\mathrm{Hk}_{\text {eff }}$ in the previous Fig. 3. Effective permeability of multilayered films formed without applied magnetic field showed much higher effective permeability at lower frequency range but rapid effective permeability drop at higher frequency range. As the annealing temperature increases effective permeability dependence of frequency for the specimen becomes smaller and, after $350{ }^{\circ} \mathrm{C}$ annealing, the effective permeability dependence of frequency becomes the same as that of the multilayered film formed under applied magnetic field. Lorentz microscope examination of the asdeposited multilayer film without magnetic field showed larger ripple than that with magnetic field. These and the $\mathrm{Hk}_{\text {eff }}$ behavior suggest that effective permeability is a strong function of $\mathrm{Hk}_{\mathrm{eff}}$ when demagnetizing field from domain structure and magnetic ripple is included as has been mentioned by Maehata et. al. ${ }^{(4)}$

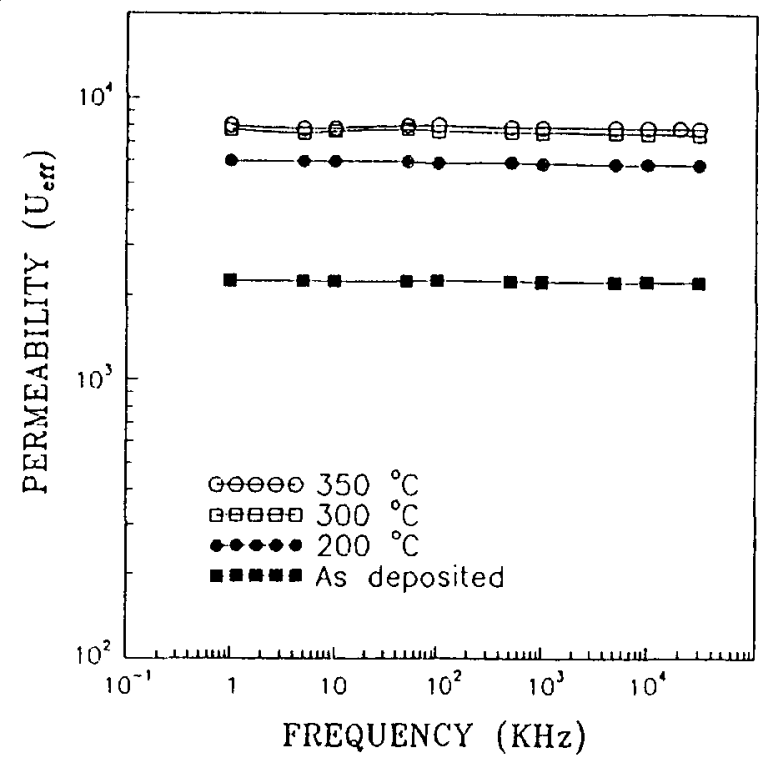

Fig. 7 Dependence of effective permeability on frequency (After RFA 2hr, hard direction, $\mathrm{SiO}_{2} 20 \mathrm{~A}, 4$ layers, $\mathrm{Hex}=600 \mathrm{mOe}$ )
Fig. 7 shows change of effective permeability response to frequency for monolithic and multilayered films after $350{ }^{\circ} \mathrm{C}$ RFA. As the number of stackings is increasing, effective permeability drop shifts to a higher frequency range. For the same number of stackings, effective permeability drop appears at lower frequency region, when the spacer thickness increases from $20 \mathrm{~A}$ to $50 \mathrm{~A}$. This indicates magnetic interaction between the layers decreases earlier for the thicker non-magnetic spacer specimens.

To study eddy current contribution, the multilayered films with $\mathrm{Cu}$ spacer were made and their effective permeability response to frequency are shown in Fig. 8. Comparing with Fig. 5, the multilayered films with $\mathrm{Cu}$ spacer show lower effective permeability for the same spacer thickness. Other magnetic properties of these films were very similar to those of the $\mathrm{SiO}_{2}$ inserted films. Although x-ray diffraction study of the films showed no crystalline peaks, electron microscopy examination of $\mathrm{CoNbZr} / \mathrm{Cu}$ showed some fine microcrystalline $\mathrm{CoNbZr}$ phases, while

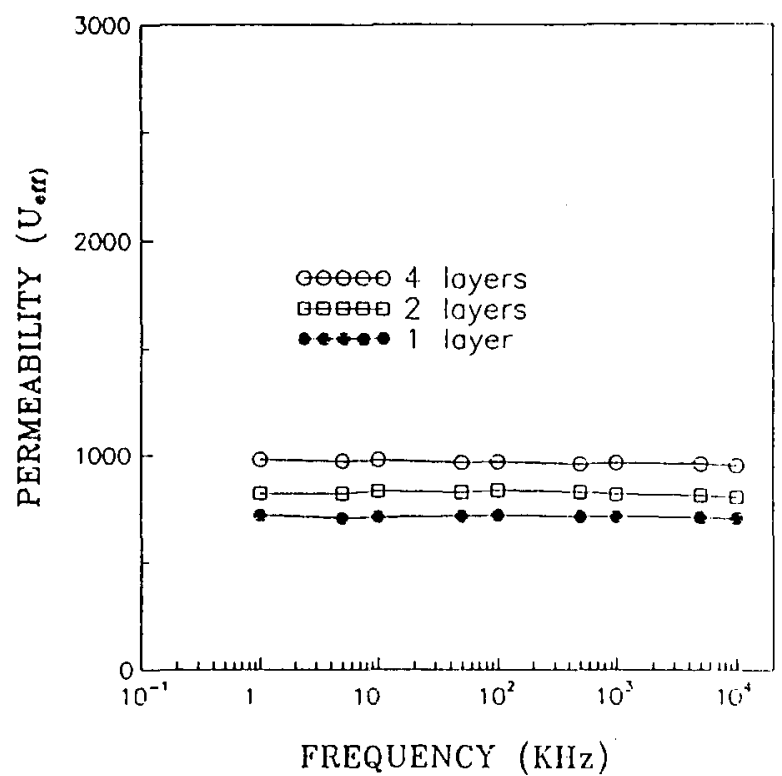

Fig. 8 Dependence of effective permeability on frequency (As-deposited, hard direction, $\mathrm{Cu}$ $50 \mathrm{~A}, \mathrm{Hex}=600 \mathrm{mOe}$ ) 
that of $\mathrm{CoNbZr} / \mathrm{SiO}_{2}$ exhibited only amorphous phase. This may be due to epitaxial growth of the $\mathrm{Co}$ alloys on the $\mathrm{Cu}$ crystalline spacer. Therefore, as a spacer an electrically conductive film with amorphous phase was necessary to eliminate this phenomenon. For the purpose a four layer stacked $\mathrm{CoNbZr} / \mathrm{Carbon}$ film was made and its effective permeability response to frequency is appeared in Fig. 9. This result is very close to that of a four layered $\mathrm{CoNbZr} / \mathrm{Cu}$. In permalloy system, frequency response of permeance for $\mathrm{NiFe}$ monolithic, $\mathrm{NiFe} / \mathrm{Zr}$ and $\mathrm{NiFe} / \mathrm{SiO}_{2}$ layers showed the same behavior up to $10 \mathrm{MHz}$ to $20 \mathrm{MHz}$ range and they concluded eddy current contribution appeared higher range than the $20 \mathrm{MHz}{ }^{(5)}$

The lower effective permeability value for $\mathrm{CoNbZr/conductive} \mathrm{spacer} \mathrm{multilayered} \mathrm{films} \mathrm{seems}$ not related to eddy current loss contribution in the frequency range we have used. However, the reason of low effective permeability throughout whole frequency range for CoNbZr/conductive spacer film is not clearly understood.

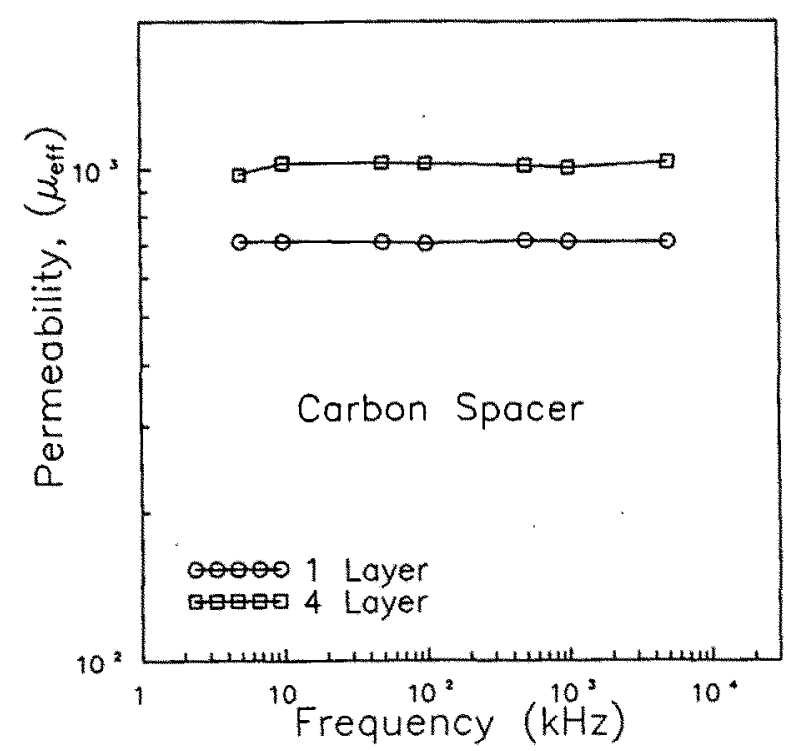

Fig. 9 Dependence of effective permeability on frequency (As-deposited, hard direction, $\mathrm{C}$ $50 \mathrm{~A}, \mathrm{Hex}=600 \mathrm{mOe}$ )

\section{Conclusion}

The followings were found by studying $\mathrm{CoNbZr}$ monolithic film and $\mathrm{CoNbZr} / \mathrm{SiO}_{2}$, $\mathrm{CoNbZr} / \mathrm{Cu}, \mathrm{CoNbZr} / \mathrm{Carbon}$ multilayered films.

1. Effective permeability value starts to drop at lower frequency range when the thickness of the dielectric layer increases from $20 \mathrm{~A}$ to $50 \mathrm{~A}$.

2. Effective anisotropy field of the films in easy direction decreases with increasing rotating field annealing temperature and becomes the same value as those of the films in hard direction after $350{ }^{\circ} \mathrm{C}$ annealing. At this point, effective permeability response of frequency becomes the same.

3. $\mathrm{CoNbZr} /$ conductive spacer scheme shows lower permeability than $\mathrm{CoNbZr} /$ dielectric spacer scheme.

\section{References}

1. Y. Shimada and N. Saito : Jap. J. of Appl. Physics, 25 (1986) 419

2. H. Fujimori, H. Morita and M. Yamamoto : IEEE Trans. Magn., 22 (1988) 1101

3. Y. Shimada and A. Hosono : J. of Mag. Soc. of Jap., 14 (1990) 397

4. Y. Maehata, S. Tsunashima and S. Uchiyama : J. of Mag. Soc. of Jap., 13 (1989) 307

5. M. Russak, C. Jahnes, M. E. Re, R. Webb and S. Mirzamaani : IEEE Trans. Magn., 26 (1990) 2332 